\title{
Intraperitoneal Fat Is Associated With Thickening of the Thoracic Aorta in Individuals at High Risk for Cardiovascular Events
}

\author{
Haroon L. Chughtai ${ }^{1}$, Timothy M. Morgan ${ }^{2}$, Craig A. Hamilton ${ }^{3}$, Charaslak \\ Charoenpanichkit ${ }^{1}$, Jingzhong Ding ${ }^{1,4,5}$, Tina E. Brinkley ${ }^{1,4,5}$, and W. Gregory Hundley ${ }^{1,6}$ \\ ${ }^{1}$ Department of Internal Medicine, Wake Forest School of Medicine, Winston-Salem, North \\ Carolina, USA \\ ${ }^{2}$ Department of Public Health Sciences, Wake Forest School of Medicine, Winston-Salem, North \\ Carolina, USA \\ ${ }^{3}$ Department of Biomedical Engineering, Wake Forest School of Medicine, Winston-Salem, North \\ Carolina, USA \\ ${ }^{4}$ Department of Gerontology, Wake Forest School of Medicine, Winston-Salem, North Carolina, \\ USA \\ ${ }^{5}$ Department of Geriatric Medicine, Wake Forest School of Medicine, Winston-Salem, North \\ Carolina, USA \\ ${ }^{6}$ Department of Radiology, Wake Forest School of Medicine, Winston-Salem, North Carolina, \\ USA
}

\begin{abstract}
Increased intraperitoneal (IP) fat is associated with increased cardiovascular (CV) risk, but mechanisms for this increase in risk are not completely established. We performed this study to assess whether IP fat is associated with ascending aortic wall thickness (AOWT), a risk factor for $\mathrm{CV}$ events. Four hundred and forty-one consecutive participants, aged 55-85 years, with risk factors for $\mathrm{CV}$ events underwent magnetic resonance measures of AOWT and abdominal fat (subcutaneous (SC) fat + IP fat). For the ascending aorta, mean wall thickness of the 4th quartile of the IP fat was higher relative to the 1st quartile $(P \leq 0.001)$. This difference persisted after accounting for SC fat $(P \leq 0.001)$, as well as age, gender, height, weight, smoking, diabetes, hypertension, low-density lipoprotein-cholesterol (LDL-C), high-density lipoprotein-cholesterol (HDL-C), and C-reactive protein (CRP) $(P<0.03)$. Elevated IP fat volume is associated with an increase in ascending AOWT, a condition that promotes $\mathrm{CV}$ events in middle aged and elderly adults.
\end{abstract}

\section{INTRODUCTION}

An abnormal increase in the thickness of the wall of the ascending thoracic aorta is associated with an abnormal increase in aortic stiffness (1,2), and identifies individuals at risk for adverse cardiovascular (CV) outcomes $(3,4)$, neurological events $(5)$, and coronary

\footnotetext{
(C) 2011 The Obesity Society

Correspondence: W. Gregory Hundley (ghundley@wfubmc.edu).

DISCLOSURE

The authors declared no conflict of interest.
} 
artery disease (CAD) (6,7). Clinical states such as advancing age, hypertension, diabetes, and hypercholesterolemia $(8,9)$ invoke increased sympathetic activity $(10)$, insulin resistance (11), vascular smooth muscle growth (12), and systemic inflammation (13). These processes contribute to enlargement of the intimal and medial layers of aortic wall resulting in an increase in ascending thoracic aortic wall thickness (AOWT).

Interestingly, increased visceral (intraperitoneal (IP)) fat is associated with adverse CV events $(3,5,14)$, and is also associated with many of the clinical conditions (diabetes) $(11,15)$, hypertension (16), hypercholesterolemia (17-19), and abnormalities (systemic inflammation and atherosclerosis $(14,20)$, that are involved in the pathophysiology of adverse $\mathrm{CV}$ events and increased AOWT. To this end, we sought to determine whether a relationship existed between IP fat volume and an increase in the mean wall thickening of the ascending aorta. To address this hypothesis, we sought to determine the relationship between IP fat and AOWT after accounting for other factors known to influence AOWT, including age, gender, height, weight, smoking, diabetes, hypertension, serum cholesterol levels and serum levels of C-reactive protein (CRP).

\section{METHODS AND PROCEDURES}

\section{Study population}

The study was approved by the institutional review board of the Wake Forest School of Medicine, and each participant provided witnessed informed consent. Four hundred and forty-one consecutive participants from the National Institutes of Health funded cohort study, "Pulmonary Edema and Stiffness of the Vascular System" (PREDICT) were enrolled. PREDICT was formulated to identify abnormalities of the vascular system that forecast future cardiac events. To accomplish this purpose, middle aged and elderly individuals (aged 55-85 years) with risk factors for a first episode of a CV event were recruited from rural communities in western North Carolina to receive a cardiac magnetic resonance (CMR) imaging exam followed by semiannual surveillance for adverse CV events. To date, PREDICT is in the first 1.5 years of participant enrollment and follow-up has yet to occur. The present analysis examines results from cross-sectional comparisons within the baseline CMR exam.

\section{Study design}

Historical, physical exam, laboratory, and CMR data were collected upon enrollment in the study. Anthropometric measurements including weight and height were performed in loose clothing without shoes. Laboratory assessments including fasting serum electrolytes, creatinine, glucose, lipids, and CRP were acquired, and then each participant underwent a CMR exam at a field strength of 1.5T (Siemens Medical Solution, Erlangen, Germany). During the CMR exam, images were acquired for the purpose of determining AOWT and abdominal fat.

For the purpose of this study, hypertension was defined according to the "Seventh report of the Joint National Committee on Prevention, Detection, Evaluation, and Treatment of High Blood Pressure" (JNC VII) as a systolic blood pressure of $\geq 140 \mathrm{~mm} \mathrm{Hg}$, a diastolic blood pressure $\searrow 90 \mathrm{~mm} \mathrm{Hg}$, or the concurrent use of antihypertensive medications (21). Diabetes was defined according to guidelines of American Diabetic Association as random plasma glucose concentration $\geq 200 \mathrm{mg} / \mathrm{dl}(11.1 \mathrm{mmol} / \mathrm{l})$ or fasting plasma glucose $\geq 126 \mathrm{mg} / \mathrm{dl}(7.0$ $\mathrm{mmol} / \mathrm{l}$ ) or a previous diagnosis of diabetes and currently on antiglycemic treatment (22). We also defined CAD in accordance with American College of Cardiology/American Heart Association guidelines (23). 
Each component of the data acquisition was accomplished by personnel blinded to other components of the study. For example, those acquiring and documenting historical, physical exam and laboratory data were blinded to the results of CMR. Those analyzing AOWT vs. abdominal fat were blinded to each other's results as well as the historical, physical exam and laboratory data: in essence this was a blinded, unpaired read.

\section{CMR assessment of the ascending thoracic aorta}

Images used to determine AOWT were acquired according to previously published techniques (24-28). Images of the thoracic aorta were acquired from an axial slice positioned $\sim 4 \mathrm{~cm}$ distal to the aortic valve (at the level of the main pulmonary artery). Imaging parameters included a prospectively ECG gated, T2 weighted, fat suppressed, breath-held, black-blood turbo-spin echo sequence using a 10-mm thick slice, a $320 \times 290$ image matrix, a $40 \mathrm{~cm}$ field of view, a $65^{\circ}$ flip angle, a 21 -ms echo train length with 8 echo trains per slice, a trigger pulse every 2 beats, a repetition time of $2 \mathrm{R}-\mathrm{R}$ intervals, and a 35 ms echo time.

We measured the maximum, minimum, and mean AOWT. Minimum AOWT represented baseline wall thickness of the aorta whereas maximum wall thickness represented focal atherosclerotic plaques (25). Measurements, performed according to previously published techniques (Figure 1) $(24,25)$, were obtained by drawing a region of interest along the inner and outer contours of the walls of ascending aorta using in-house software developed in Matlab (The Mathworks, Natick, MA). Images were magnified by a factor of 8 to provide subpixel measurement of area. Radial lines were drawn circumferentially between the outer and inner regions of interests. The length of these radial lines was measured in $\mathrm{mm}$.

Maximum length represented maximum wall thickness and minimum length represented minimum wall thickness. The mean length in $\mathrm{mm}$ was the average length of all the radial lines around the circumference of the aorta (Figure 1).

\section{CMR measurement of abdominal fat}

Total and compartmental amounts of abdominal fat were determined from an axial slice positioned at the level of the second lumbar vertebra according to previously published techniques (29). As shown in Figure 2, we divided abdominal fat into subcutaneous (SC) fat, and IP fat compartments using the SliceOmatic 4.2 Rev-10 software program (Tomovision, Montreal, Canada) (30). Adipose tissue was separated from other tissues based on pixel intensity. Different tissues were segmented and stained initially with different colors using an automated algorithm in the SliceOmatic software (morpho mode) based on the difference of the pixel intensities. These drawings were then adjusted and corrected using manual tools. To calculate compartmental fat area, the number of subpixels within each fat compartment (SC and IP) was multiplied by the size of subpixels. This area was then multiplied by the slice thickness to determine the volume of fat $\left(\mathrm{in}^{3}\right)$ in each compartment. To determine the interobserver variability of the measurements of fat within each compartment, images from 20 randomly selected participants were redrawn by a different observer (CC) blinded to all other study results.

\section{Statistical analysis}

Due to skewness in distribution, certain variables were converted into logarithmic scales (AOWTs, CRP, glucose, cholesterol, low-density lipoprotein-cholesterol (LDL-C) and triglycerides), and other variables were converted into square roots (IP and SC fat measures). Statistical tests to determine whether the continuous increasing levels of IP fat were associated with increasing or decreasing levels of other factors were based on correlation coefficients (using square roots and logs where appropriate). Groups were created by the quartiles ( $25 \%$ each) of IP fat in order to illustrate trends. Stepwise multiple 
regressions were used to adjust the association between fat volumes and aortic measurements that may account for the relationship between fat volumes and aortic measurements. Factors that were considered as potential confounders include: age, gender, height, weight, BMI, smoking, diabetes, hypertension, hypercholesterolemia, CAD, CRP, and aortic diameter. A potential confounder was included in the model if it was significantly independently associated with wall thickness at the $10 \%$ level of significance. To compare dichotomous variables of fat volume, 2 -sample independent $X^{2}$ was used with IP fat as a dependant variable. Unless stated otherwise, all data were presented as mean \pm s.d.; a twosided $P$ value $<0.05$ was considered significant. The authors had full access to the data and take responsibility for its integrity. All authors have read and agree to the manuscript as written.

\section{RESULTS}

The baseline clinical and laboratory characteristics of the study participants according to quartiles of IP fat are shown in Table 1. Subjects with larger amounts of IP fat were more likely to be men, taller $(P<0.001$ for both), diabetic $(P=0.03)$, and exhibit hypercholesterolemia $(P=0.03)$. Similarly, comparing individuals in the 4 th quartile of the IP fat with those in the 1st quartile, participants in the 4th quartile exhibited higher serum levels of triglycerides (mean of $1.86 \mathrm{vs.} 1.15 \mathrm{mmol} / \mathrm{l}$ (165 vs. $102 \mathrm{mg} / \mathrm{dl}$ ), $P \leq 0.001$ ), fasting glucose (mean of 6.88 vs. $5.94 \mathrm{mmol} / \mathrm{l}(124 \mathrm{vs.} 107 \mathrm{mg} / \mathrm{dl}$ ), $P=0.005)$, LDL-C (mean of 2.97 vs. $2.64 \mathrm{mmol} / \mathrm{l}$ (115 vs. $102 \mathrm{mg} / \mathrm{dl}$ ), $P=0.03$ ), CRP (mean of $35 \mathrm{vs.} 22 \mathrm{mg} / \mathrm{l}$ ( $3.5 \mathrm{vs}$. $2.2 \mathrm{mg} / \mathrm{dl}$ ), $P=0.22$ ), and lower levels of high-density lipoprotein-cholesterol (HDL-C) (mean of $1.01 \mathrm{vs.} 1.45 \mathrm{mmol} / \mathrm{l}$ (39 vs. $56 \mathrm{mg} / \mathrm{dl}$ ), $P \leq 0.001$ ). IP fat also correlated with age $(r=0.28, P<0.001)$.

CMR measurements of AOWT are shown in Table 2. For the ascending aorta, minimum, maximum, and mean wall thickness of the 4th quartile of IP fat was higher relative to the 1st quartile ( $P \leq 0.001$ for all). As shown in Table 3 , this association between IP fat volume and AOWT persisted after adjusting for SC fat. Unlike IP fat, SC fat demonstrated a borderline correlation with AOWT $(r=0.09,0.09$, and 0.10 , respectively for mean, minimum, and maximum AOWT $(P=0.06,0.06$, and 0.04 respectively $)$ ). However, after adjusting for IP fat, this association was lost (Table 3). We performed a multiple regression analysis to determine if the association between IP fat and AOWT was influenced by other conditions associated with abnormal increases in AOWT. Factors that were considered as potential confounding factors were age, gender, height, weight, smoking, diabetes, hypertension, LDL-C, HDL-C, CRP, and aortic diameter. Stepwise regression was used to select those factors that were significantly associated with wall thickness. The significant possible confounders that were adjusted in the model were $\operatorname{CAD}(P=0.004)$ and $\operatorname{CRP}(P=0.026)$ for mean AOWT, diabetes $(0.016$ and CAD 0.011) for minimum AOWT, CAD $(P=0.046)$ for maximum AOWT. The association between IP fat and mean, minimum, and maximum AOWT persisted (Table 4). In regression models containing IP fat and aortic diameter, IP fat remained significantly associated with mean, minimum, and maximum AOWT $(P<0.001, P$ $<0.001$, and $P<0.001$, respectively); whereas, aortic diameter was not independently associated with mean, minimum, or maximum AOWT ( $P=0.21, P=0.82$, and $P=0.08$, respectively). When IP fat and BMI were both included in models for predicting AOWT, IP fat remained a strong independent predictor of mean, minimum, and maximum AOWT $(P<$ 0.001 for all); however, once IP fat was in the model, BMI showed no association with mean, minimum, and maximum AOWT ( $P=0.92, P=0.94$, and $P=46$, respectively).

The association of IP fat with mean AOWT was evaluated in an analysis stratified on gender. There was no significant interaction with gender, and the associations between IP fat 
and AOWT remained significant within each gender (men, $r=0.29, P<0.001$; women, $r=$ $0.15, P=0.03$ for mean AOWT).

Sleep apnea can be present in obese patients. In our population nine $(2.2 \%)$ of the patients had sleep apnea. The AOWT was greater for those patients with sleep apnea $P<0.001, P<$ 0.001 , and $P=0.020$ for mean, minimum, and maximum, respectively. When sleep apnea was added to IP fat in the model predicting mean AOWT, both variables were independent predictors of mean AOWT with adjusted $P$ values of $P<0.001$ for IP fat and $P=0.003$ for sleep apnea.

As age increased in this cohort of individuals at high risk for cardiac events, mean AOWT also increased $(r=0.22, P<0.001$; Figure 3$)$. Ascending AOWT averaged $2.72 \mathrm{~mm}$ in those $\checkmark 65$ years of age, vs. $2.99 \mathrm{~mm}$ in those $>65$ years of age $(P<0.001)$. We sought to determine whether those participants with an increase in IP fat exhibited an increase in mean AOWT relative to similarly aged individuals without an increase in IP fat. As shown in Figure 3, mean AOWT was significantly higher in those aged $\$ 65$ years with IP fat $>$ median value v2imilarly aged individuals with IP fat $\leq$ median value $(P<0.001)$. These findings were also present for those $>65$ years in age $(P<0.005)$. In individuals $\$ 55$ years of age, high IP fat was associated with a mean AOWT that was similar in magnitude to the wall thickness observed in individuals $>65$ years of age with low IP fat $(P=0.587$ for difference between the groups).

\section{DISCUSSION}

The results of this study indicate that in middle aged and elderly patients at risk for CV events: (i) IP fat is associated with increased AOWT of the ascending thoracic aorta (an abnormal increase in minimum and mean AOWT); (ii) this association persists after accounting for age, height, gender, weight, smoking, diabetes, hypertension, LDL-C, HDL$\mathrm{C}$, and CRP (CV risk factors associated previously with an increase in AOWT); and (iii) an increased amount of IP fat in individuals $\$ 65$ years of age is associated with wall thickening in the ascending thoracic aorta that is similar in magnitude to that observed in individuals $>65$ years of age without an increase in IP fat. To the best of our knowledge, this is the first study to show the relationship between IP fat and ascending AOWT (an area remote to the abdomen) after accounting for age, gender, weight, height, and other established CV risk factors.

We used CMR for assessment of AOWT because it has been validated both in animals (26) and humans (27) for assessment of aortic wall morphology and thickness. The technique we used incorporates fat saturation so that signal from surrounding fat does not interfere with the assessment of AOWT (28). Our IP fat and AOWT measurements were comparable to previous studies. Mean and maximum AOWTs in our study were $2.90 \mathrm{~mm}$ and $3.84 \mathrm{~mm}$, compared to $2.35 \mathrm{~mm}$ and $3.51 \mathrm{~mm}$, respectively from a previous study of 1,053 individuals without overt CAD from the Multiethnic Study of Atherosclerosis (2). SC and IP fats in our study were $219 \mathrm{~cm}^{3}$ and $138 \mathrm{~cm}^{3}$ compared to $150 \mathrm{~cm}^{3}$ and $125 \mathrm{~cm}^{3}$, respectively, from a previous study of 49 men without any clinical disease taking no medications with an average BMI of 28 (range 21-46) (29).

We divided IP fat into quartiles only in order to illustrate trends and not for hypothesis testing. As shown in Table 1, people in the 4th quartile of IP fat were more likely to be older, of male gender, with an increased BMI, and possess a higher prevalence of diabetes, hypertension, and smoking. Similarly, people in the 4th quartile of IP fat exhibited a more unfavorable lipid profile as compared to the 1st quartile (higher LDL-C and triglycerides 
with lower HDL-C). Minimum, maximum, and mean AOWT increased progressively from 1st quartile to 4 th quartile (Table 2).

To determine whether the variables that displayed differences across quartiles shown in Table 1 could have influenced the association of AOWT and IP fat, we performed multiple linear regression analyses incorporating these variables in the model. As shown in Table 4, IP fat volume remained independently associated with mean and minimum AOWT after accounting for these variables; whereas maximal wall thickness did not. Minimum and mean AOWT (as opposed to maximum AOWT) may represent diffuse intimal thickening related to early atherosclerosis, vascular inflammation, or increased vascular smooth muscle growth that may not result in significant luminal narrowing $(31,32)$. Maximum AOWT, on the other hand, may represent eccentric focal plaque formation associated with advanced atherosclerosis. This relatively large plaque formation has been associated with risk factors for CAD, including for example hypercholesterolemia. Similar to prior studies, in our study, age correlated with ascending AOWT (33). As shown in Figure 3, in individuals $\mathbf{6 5}$ years of age, high IP fat was associated with an AOWT that was similar in magnitude to the amount that was present in individuals $>65$ years of age but with low IP fat. These data raise important questions to address in future studies. For example, does increased IP fat in younger individuals create a situation in the aorta that is similar to that observed in an elderly person's aorta without increased IP fat? Could this "premature thickening" explain potential mechanisms for increased adverse $\mathrm{CV}$ outcomes in obese young individuals previously shown in epidemiological studies (34).

There are several reasons why IP fat may be associated with AOWT. First, insulin resistance accompanies IP or visceral adiposity (11). Insulin may promote sodium reabsorption, increased sympathetic activity (10), and vascular smooth muscle growth (12). All these factors are associated with increased vascular wall thickness. Second, high plasma glucose may promote advanced glycation end products which deposit in the vessel wall, crosslink collagen, and thereby thicken and stiffen the arterial wall (35). Third, obese individuals with more IP fat often exhibit a low-grade inflammatory state which may lead to continuous vascular inflammation (36) and increased growth of the intimal-medial layer of arterial segments (37). Laboratory data of our participants supported these mechanisms as glucose, LDL-C, triglycerides, and CRP increased and HDL-C decreased from 1st quartile to the 4th quartile of IP fat in our results.

Our results have important clinical implications. Ascending AOWT is linked to adverse CV outcomes (3-7), decreased coronary flow reserve (1), and aortic stiffness (38), a determinant of left ventricular hypertrophy. Our observed strong independent associations of IP fat with AOWT suggests further studies should be performed to establish whether there are functional consequences of IP fat on left ventricular performance that are mediated through an increase in ascending aortic wall thickening. In addition, studies performed to reduce visceral or IP fat may want to include AOWT as a surrogate end point.

Our study has limitations. First, our cross-sectional study design does not allow us to establish a causal relationship between IP fat and AOWT. We were, however, able to demonstrate an important association between IP fat and AOWT after accounting for established causes of increased wall thickness. Second, our study population was primarily white $(90 \%)$, thus we are uncertain of the effects of visceral fat on the AOWT in individuals of various race and ethnicity. Finally, our PREDICT participants included those at high risk for adverse $\mathrm{CV}$ events. Further data from older individuals without $\mathrm{CV}$ risk factors would be necessary to examine the relationship in the healthy population without these risk factors. 
In conclusion, after accounting for other factors known to influence vascular wall thickening, IP fat depots are independently associated with wall thickening of the aorta, a stimulus for LV hypertrophy and a marker for adverse CV events.

\section{Acknowledgments}

This research was supported in part by the following grants from The National Institute of Health: ROIHL076438, R33CA1219601, and P30AG21332.

\section{References}

1. Nemes A, Forster T, Csanády M, Gruber N. Indices of aortic distensibility and coronary flow velocity reserve in patients with different grades of aortic atherosclerosis. Int J Cardiovasc Imaging. 2004; 20:271-277. [PubMed: 15529908]

2. Malayeri AA, Natori S, Bahrami H, et al. Relation of aortic wall thickness and distensibility to cardiovascular risk factors (from the Multi-Ethnic Study of Atherosclerosis [MESA]). Am J Cardiol. 2008; 102:491-496. [PubMed: 18678312]

3. Schachner T, Zimmer A, Nagele G, et al. The influence of ascending aortic atherosclerosis on the long-term survival after CABG. Eur J Cardiothorac Surg. 2005; 28:558-562. [PubMed: 16126401]

4. Amanullah AM, Artel BJ, Grossman LB, Espioneza A, Chaudhry FA. Usefulness of complex atherosclerotic plaque in the ascending aorta and arch for predicting cardiovascular events. Am J Cardiol. 2002; 89:1423-1426. [PubMed: 12062741]

5. van der Linden J, Bergman P, Hadjinikolaou L. The topography of aortic atherosclerosis enhances its precision as a predictor of stroke. Ann Thorac Surg. 2007; 83:2087-2092. [PubMed: 17532403]

6. Frogoudaki A, Barbetseas J, Aggeli C, et al. Thoracic aorta atherosclerosis burden index predicts coronary artery disease in patients undergoing transesophageal echocardiography. Atherosclerosis. 2008; 197:232-236. [PubMed: 17524407]

7. Nemes A, Thury A, Forster T, Boda K, Csanády M. Grade of aortic atherosclerosis: a valuable adjunct to coronary flow velocity reserve in the evaluation of coronary artery disease. Ups J Med Sci. 2007; 112:73-82. [PubMed: 17578810]

8. Oyama N, Gona P, Salton CJ, et al. Differential impact of age, sex, and hypertension on aortic atherosclerosis: the Framingham Heart Study. Arterioscler Thromb Vasc Biol. 2008; 28:155-159. [PubMed: 17991874]

9. Bozkurt A, Cayli M, Demir M, Alhan C, Acartürk E. The relation between aortic atherosclerosis and risk factors. Anadolu Kardiyol Derg. 2007; 7:2-5. [PubMed: 17347066]

10. Young JB. Effect of experimental hyperinsulinemia on sympathetic nervous system activity in the rat. Life Sci. 1988; 43:193-200. [PubMed: 3292869]

11. Bonadonna RC, Groop L, Kraemer N, et al. Obesity and insulin resistance in humans: a doseresponse study. Metab Clin Exp. 1990; 39:452-459. [PubMed: 2186255]

12. Begum N, Song Y, Rienzie J, Ragolia L. Vascular smooth muscle cell growth and insulin regulation of mitogen-activated protein kinase in hypertension. Am J Physiol. 1998; 275:C42C49. [PubMed: 9688833]

13. Park HS, Park JY, Yu R. Relationship of obesity and visceral adiposity with serum concentrations of CRP, TNF-alpha and IL-6. Diabetes Res Clin Pract. 2005; 69:29-35. [PubMed: 15955385]

14. Nicklas BJ, Penninx BW, Cesari M, et al. Health, Aging and Body Composition Study. Association of visceral adipose tissue with incident myocardial infarction in older men and women: the Health, Aging and Body Composition Study. Am J Epidemiol. 2004; 160:741-749. [PubMed: 15466496]

15. Sparrow D, Borkan GA, Gerzof SG, Wisniewski C, Silbert CK. Relationship of fat distribution to glucose tolerance. Results of computed tomography in male participants of the Normative Aging Study. Diabetes. 1986; 35:411-415. [PubMed: 3956878]

16. Kanai H, Matsuzawa Y, Kotani K, et al. Close correlation of intra-abdominal fat accumulation to hypertension in obese women. Hypertension. 1990; 16:484-490. [PubMed: 2228147] 
17. Després JP, Moorjani S, Lupien PJ, et al. Regional distribution of body fat, plasma lipoproteins, and cardiovascular disease. Arteriosclerosis. 1990; 10:497-511. [PubMed: 2196040]

18. Nguyen-Duy TB, Nichaman MZ, Church TS, Blair SN, Ross R. Visceral fat and liver fat are independent predictors of metabolic risk factors in men. Am J Physiol Endocrinol Metab. 2003; 284:E1065-E1071. [PubMed: 12554597]

19. Banerji MA, Buckley MC, Chaiken RL, et al. Liver fat, serum triglycerides and visceral adipose tissue in insulin-sensitive and insulin-resistant black men with NIDDM. Int J Obes Relat Metab Disord. 1995; 19:846-850. [PubMed: 8963350]

20. Lear SA, Humphries KH, Kohli S, et al. Visceral adipose tissue, a potential risk factor for carotid atherosclerosis: results of the Multicultural Community Health Assessment Trial (M-CHAT). Stroke. 2007; 38:2422-2429. [PubMed: 17673711]

21. Chobanian AV, Bakris GL, Black HR, et al. Joint National Committee on Prevention, Detection, Evaluation, and Treatment of High Blood Pressure. National Heart, Lung, and Blood Institute; National High Blood Pressure Education Program Coordinating Committee. Seventh report of the Joint National Committee on Prevention, Detection, Evaluation, and Treatment of High Blood Pressure. Hypertension. 2003; 42:1206-1252. [PubMed: 14656957]

22. The Expert Committee on the Diagnosis and Classification of Diabetes Mellitus: Follow-up report on the diagnosis of diabetes mellitus. Diabetes Care. 2003; 26:3160-3167. [PubMed: 14578255]

23. Fraker TD Jr, Fihn SD, Gibbons RJ, et al. American College of Cardiology; American Heart Association; American College of Cardiology/American Heart Association Task Force on Practice Guidelines Writing Group. 2007 chronic angina focused update of the ACC/AHA 2002 Guidelines for the management of patients with chronic stable angina: a report of the American College of Cardiology/American Heart Association Task Force on Practice Guidelines Writing Group to develop the focused update of the 2002 Guidelines for the management of patients with chronic stable angina. Circulation. 2007; 116:2762-2772. [PubMed: 17998462]

24. Fayad ZA, Nahar T, Fallon JT, et al. In vivo magnetic resonance evaluation of atherosclerotic plaques in the human thoracic aorta: a comparison with transesophageal echocardiography. Circulation. 2000; 101:2503-2509. [PubMed: 10831525]

25. Herrington DM, Brown WV, Mosca L, et al. Relationship between arterial stiffness and subclinical aortic atherosclerosis. Circulation. 2004; 110:432-437. [PubMed: 15262851]

26. Helft G, Worthley SG, Fuster V, et al. Atherosclerotic aortic component quantification by noninvasive magnetic resonance imaging: an in vivo study in rabbits. J Am Coll Cardiol. 2001; 37:1149-1154. [PubMed: 11263622]

27. Corti R, Fayad ZA, Fuster V, et al. Effects of lipid-lowering by simvastatin on human atherosclerotic lesions: A longitudinal study by high-resolution, noninvasive magnetic resonance imaging. Circulation. 2001; 104:249-252. [PubMed: 11457739]

28. Corti R, Fuster V, Fayad ZA, et al. Lipid lowering by simvastatin induces regression of human atherosclerotic lesions: two years' follow-up by high-resolution noninvasive magnetic resonance imaging. Circulation. 2002; 106:2884-2887. [PubMed: 12460866]

29. Abate N, Garg A, Coleman R, Grundy SM, Peshock RM. Prediction of total subcutaneous abdominal, intraperitoneal, and retroperitoneal adipose tissue masses in men by a single axial magnetic resonance imaging slice. Am J Clin Nutr. 1997; 65:403-408. [PubMed: 9022523]

30. Bonekamp S, Ghosh P, Crawford S, et al. Quantitative comparison and evaluation of software packages for assessment of abdominal adipose tissue distribution by magnetic resonance imaging. Int J Obes (Lond). 2008; 32:100-111. [PubMed: 17700582]

31. Ross R, Glomset JA. The pathogenesis of atherosclerosis (second of two parts). N Engl J Med. 1976; 295:420-425. [PubMed: 778621]

32. Nakashima Y, Wight TN, Sueishi K. Early atherosclerosis in humans: role of diffuse intimal thickening and extracellular matrix proteoglycans. Cardiovasc Res. 2008; 79:14-23. [PubMed: 18430750]

33. Marque V, Kieffer P, Atkinson J, Lartaud-Idjouadiene I. Elastic properties and composition of the aortic wall in old spontaneously hypertensive rats. Hypertension. 1999; 34:415-422. [PubMed: 10489387] 
34. Franks PW, Hanson RL, Knowler WC, et al. Childhood obesity, other cardiovascular risk factors, and premature death. N Engl J Med. 2010; 362:485-493. [PubMed: 20147714]

35. Henry RM, Kostense PJ, Spijkerman AM, et al. Hoorn Study. Arterial stiffness increases with deteriorating glucose tolerance status: the Hoorn Study. Circulation. 2003; 107:2089-2095. [PubMed: 12695300]

36. Fontana L, Eagon JC, Trujillo ME, Scherer PE, Klein S. Visceral fat adipokine secretion is associated with systemic inflammation in obese humans. Diabetes. 2007; 56:1010-1013. [PubMed: 17287468]

37. Thalmann S, Meier CA. Local adipose tissue depots as cardiovascular risk factors. Cardiovasc Res. 2007; 75:690-701. [PubMed: 17412312]

38. Zureik M, Bureau JM, Temmar M, et al. Echogenic carotid plaques are associated with aortic arterial stiffness in subjects with subclinical carotid atherosclerosis. Hypertension. 2003; 41:519_ 527. [PubMed: 12623953] 


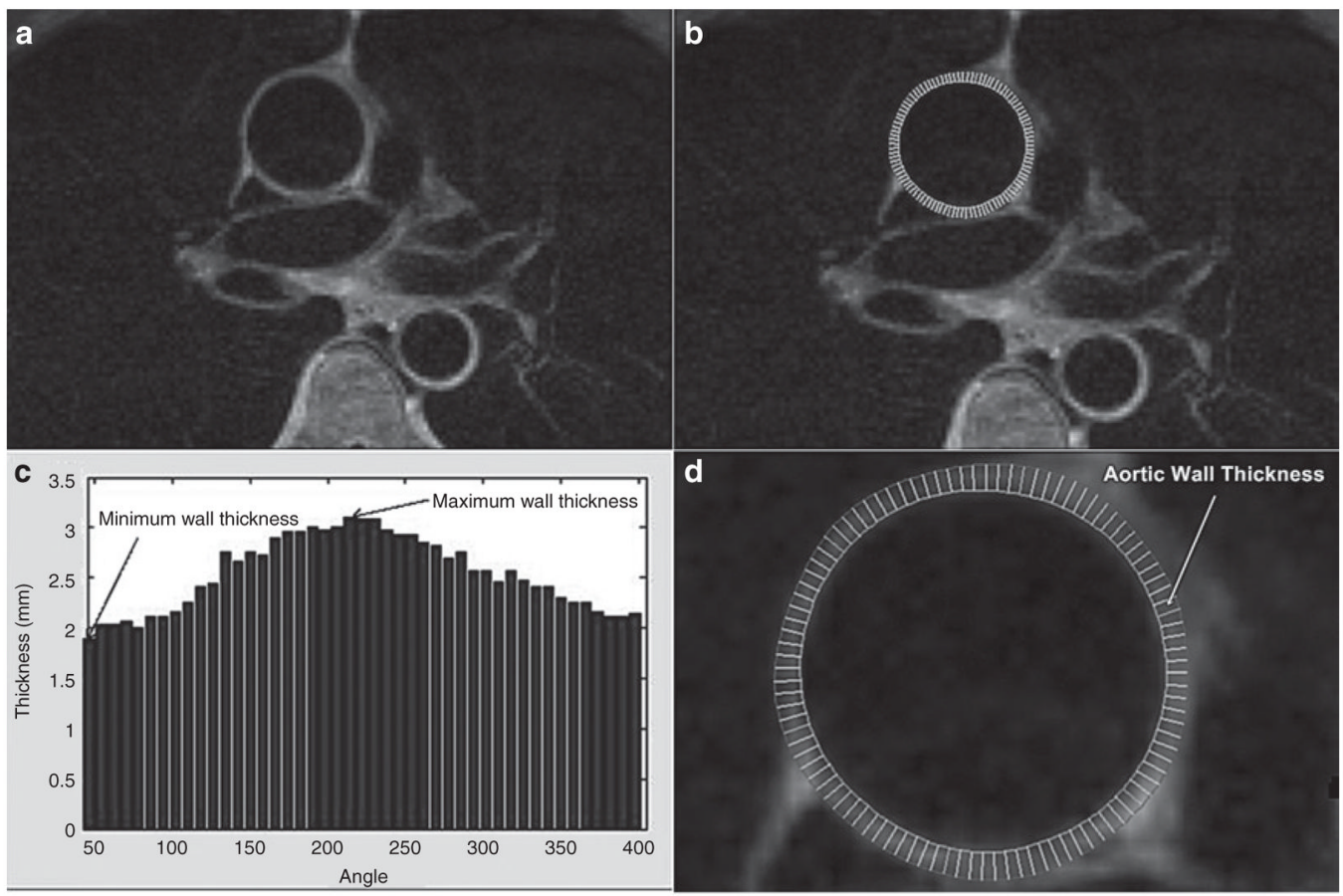

Figure 1.

Method to determine aortic wall thickness (AOWT) (aortic wall thickness), (a) before and (b) after drawing contours (outer and inner). (c) The AOWT distribution with wall thickness in mm plotted on the $y$-axis and angle from the center of the lumen on the $\mathrm{x}$-axis (maximum and minimum AOWT are shown). (d) Magnified drawing of wall thickness in which the white radii are used to calculate the minimum, maximum, and mean wall thickness. 


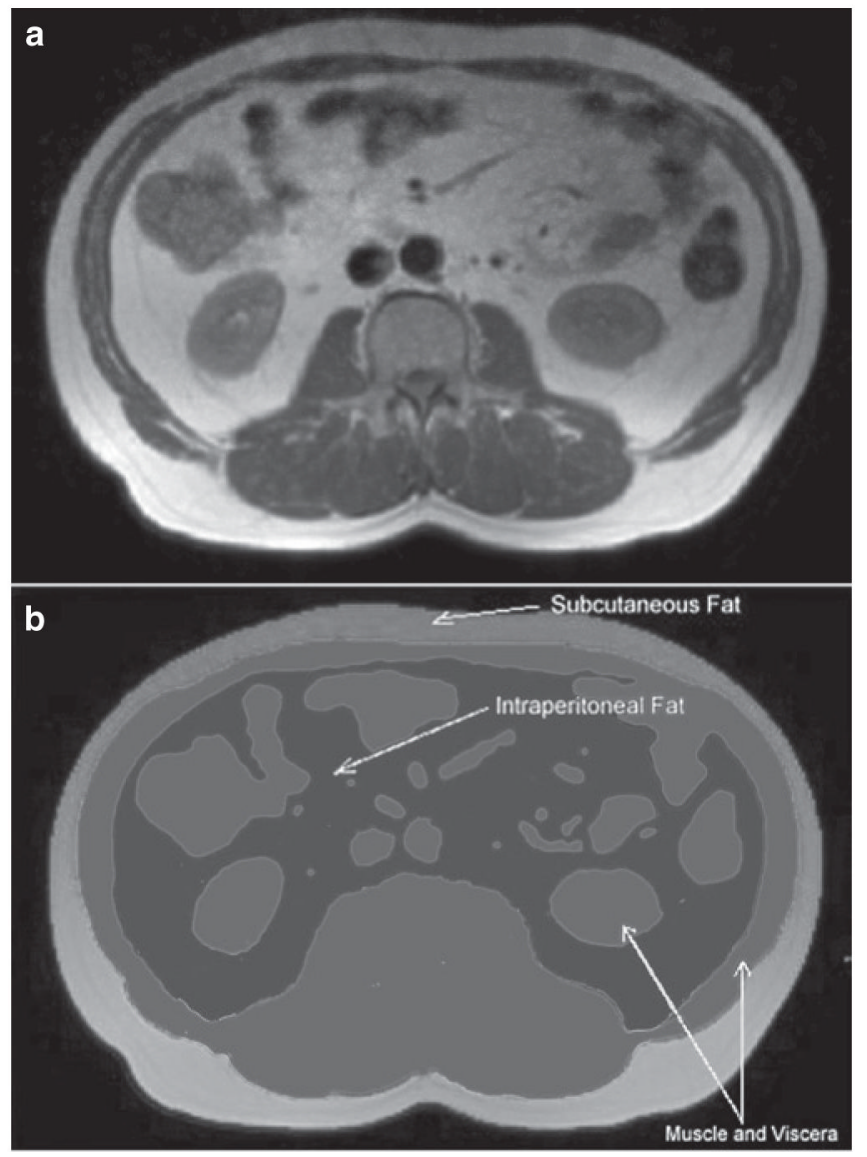

Figure 2.

Identification of fat depots. (a) An axial slice used for quantitation of fat and (b) highlights color shadowing used to segment the abdominal slice into visceral organs and fat depots. Voxels identified in white represent subcutaneous fat, those in black demarcate intraperitoneal fat. Gray voxels identify muscular tissue and viscera. 


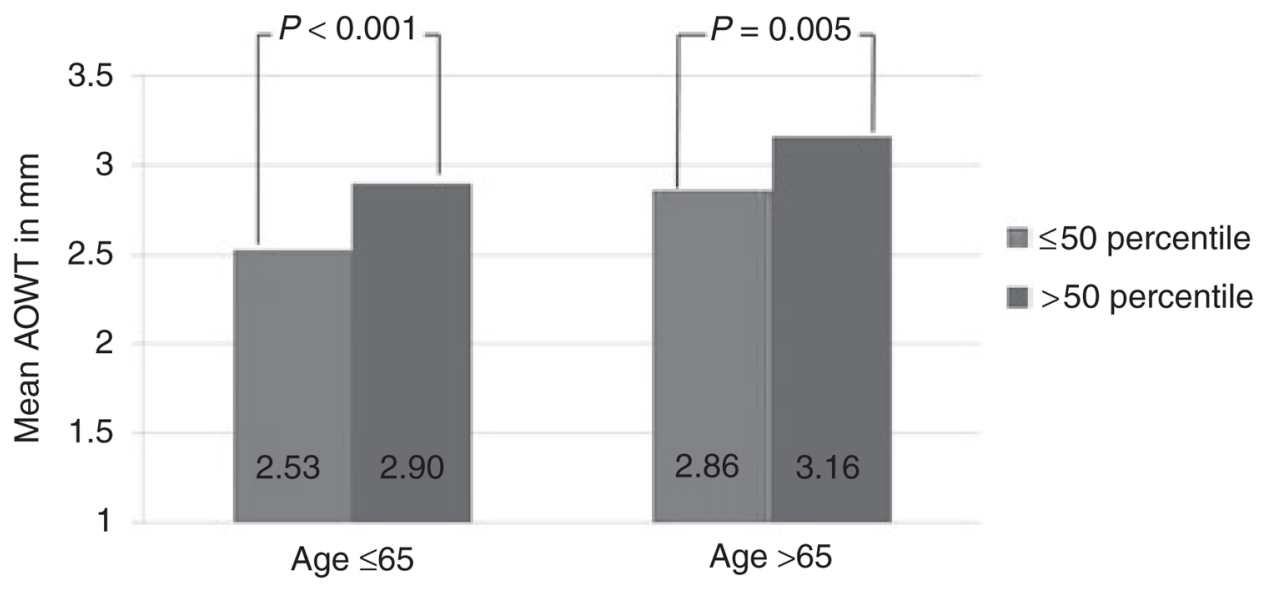

Figure 3.

Mean ascending aortic wall thickness stratified by age (below and above 65 years). Light gray bars represent those with intraperitoneal fat $\mathbf{5 0 t h}$ percentile for the respective age group and dark gray bars demonstrate intraperitoneal fat $>50$ th percentile for the respective age group. 


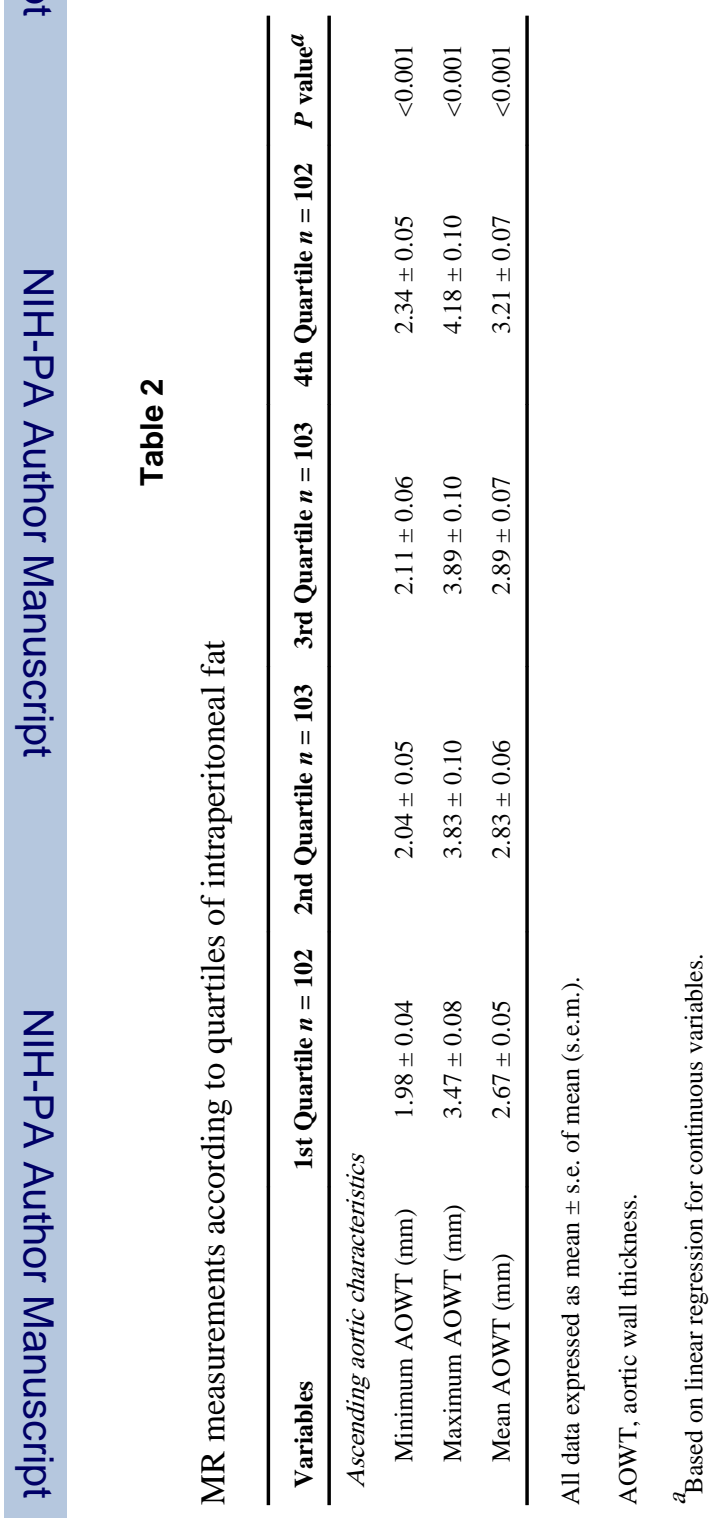

Obesity (Silver Spring). Author manuscript; available in PMC 2013 October 31. 


\section{Table 3}

Correlation of IP and SC with AOWT after adjusting for each other

\begin{tabular}{llll}
\hline & $\begin{array}{l}\text { Minimum AOWT Correlation } \\
(\boldsymbol{P} \text { value })\end{array}$ & $\begin{array}{l}\text { Maximum AOWT Correlation } \\
(\boldsymbol{P} \text { value })\end{array}$ & $\begin{array}{l}\text { Mean AOWT Correlation }(\boldsymbol{P} \\
\text { value })\end{array}$ \\
\hline IP fat & $0.24(<0.001)$ & $0.23(<0.001)$ & $0.27(<0.001)$ \\
SC fat & $0.09(0.06)$ & $0.10(0.03)$ & $0.09(0.06)$ \\
IP fat after adjusting for SC fat & $0.23(<0.001)$ & $0.22(<0.001)$ & $0.26(<0.001)$ \\
SC fat after adjusting for IP fat & $0.05(0.24)$ & $0.07(0.16)$ & $0.05(0.28)$ \\
\hline
\end{tabular}

AOWT, aortic wall thickness; IP, intraperitoneal; SC, subcutaneous. 
Table 4

Multiple regression for intraperitoneal fat with aortic parameters

\begin{tabular}{lllll}
\hline & Correlation & Unadjusted $\boldsymbol{P}$ values & Partial correlation & Adjusted $^{\boldsymbol{a}} \boldsymbol{P}$ values \\
\hline Minimum AOWT $^{b}$ & 0.24 & $<0.001$ & 0.30 & $<0.001$ \\
Maximum AOWT & 0.23 & $<0.001$ & 0.21 & $<0.001$ \\
Mean AOWT & 0.27 & $<0.001$ & 0.25 & $<0.001$ \\
\hline
\end{tabular}

a Minimum AOWT adjusted for diabetes and CAD. Maximum AOWT adjusted for CAD and DM. Mean AOWT adjusted for CAD and CRP.

${ }^{b}$ AOWT $=$ aortic wall thickness (after substituting BMI for height, and weight resulting $P$ values were $0.001,0.17$, and 0.008 for minimum, maximum, and mean AOWT). 